Supporting Information

\title{
Tailoring Surface Properties of Electrode for Synchronous Enhanced Extracellular Electron Transfer and Enriched Exoelectrogens in Microbial Fuel Cells
}

Yunfei Li ${ }^{a}$, Jia Liu ${ }^{a^{*}}$, Xuepeng Chen ${ }^{a}$, Jingxuan Wu ${ }^{a}$, Nan Li $^{a}$, Weihua He ${ }^{a, b}$, Yujie Feng, ${ }^{a *}$

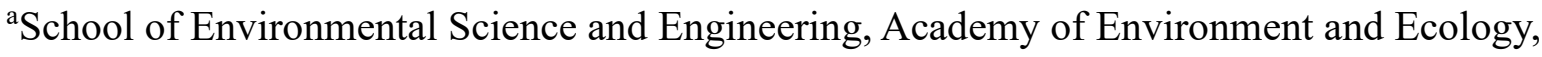

Tianjin University, No. 92 Weijin Road, Nankai District, Tianjin, 300072, China

${ }^{\mathrm{b}}$ State Key Laboratory of Urban Water Resource and Environment, Harbin Institute of

Technology, No. 73 Huanghe Road, Nangang District, Harbin, 150090, China

*Corresponding Author:

E-mail: jialiu1@tju.edu.cn; yujief@hit.edu.cn 


\section{Analysis of electrochemical dynamic behaviors of different bioanodes under non- turnover condition}

The heterogeneous electron transfer rate constant $\left(k_{\mathrm{s}}\right)$ was estimated from variation of peak potentials with scan rate according to Loviron's theory. ${ }^{1}$ The $k_{\mathrm{s}}$ was calculated by the equation:

$$
\lg k_{s}=\alpha \lg (1-\alpha)+(1-\alpha) \lg \alpha-\lg \frac{R T}{n F v}-\alpha(1-\alpha) \frac{n F \Delta E_{p}}{2.3 R T}
$$

where $\alpha$ refers to charge transfer coefficient; $\Delta E_{p}$ is the potential difference between anodic and cathodic peak; $n$ is the number of transferred electrons; $F$ represents Faraday's constant (96 $\left.487 \mathrm{C} \mathrm{mol}^{-1}\right) ; R$ is the gas constant $\left(8.314 \mathrm{~J} \mathrm{~mol}^{-1} \mathrm{~K}^{-1}\right) ; T$ is the temperature $(303 \mathrm{~K}) ; v$ is the scan rate.

The peak current $(i)$ at a fixed scan rate is composed of two terms related to the scan rate $(v):^{2}$

$$
i=k_{1} v+k_{2} v^{0.5}
$$

where $i$ is extracted from the CV curves at a fixed scan rate, $k_{1}$ and $k_{2}$ are constants. $k_{1} v$ and $k_{2} v^{0.5}$ correspond to the current contributions from the fast kinetic process and show kinetic process, respectively.

Dividing $v^{0.5}$ into both sides of Equation (2) gives:

$$
i v^{-0.5}=k_{1} v^{0.5}+k_{2}
$$

By plotting $i v^{-0.5}$ as a function of $v^{0.5}$, we can determine $k_{1}$ and $k_{2}$. Then the current contribution ratios from the fast kinetic process and show kinetic process were obtained at each scan rate. 

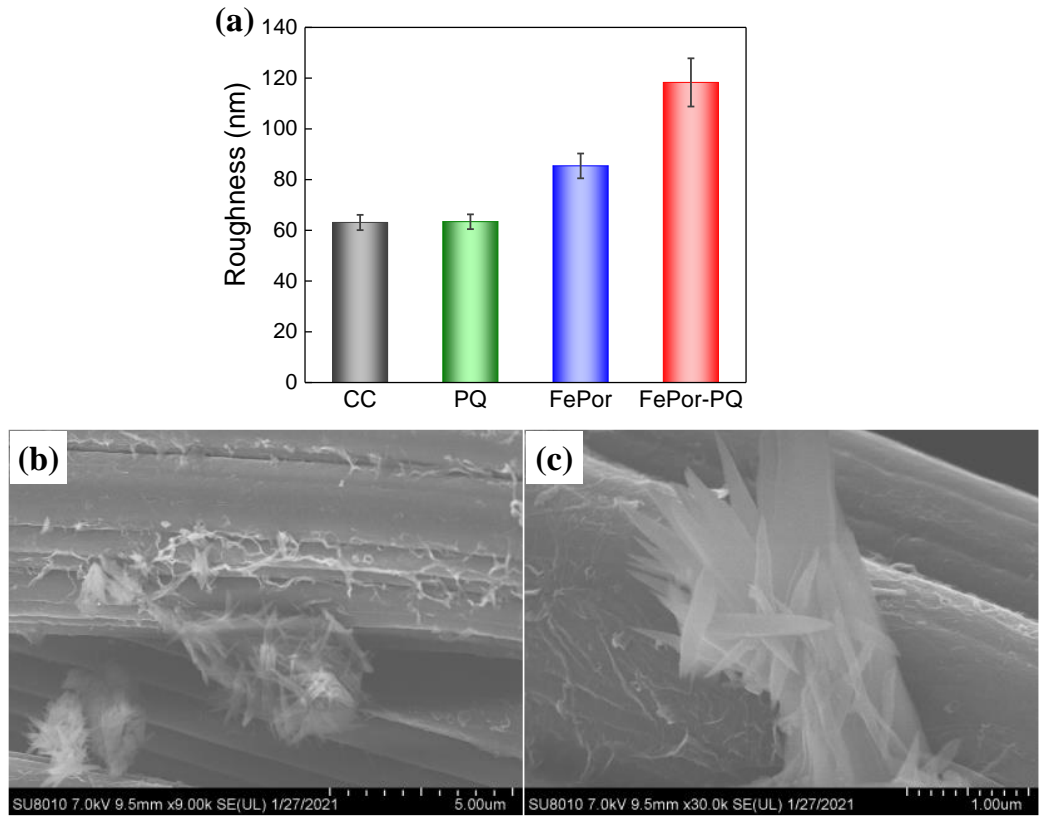

Figure S1. (a) The roughness of CC, PQ, FePor, and FePor-PQ electrodes; (b) and (c), SEM images of FePor-PQ electrodes. 

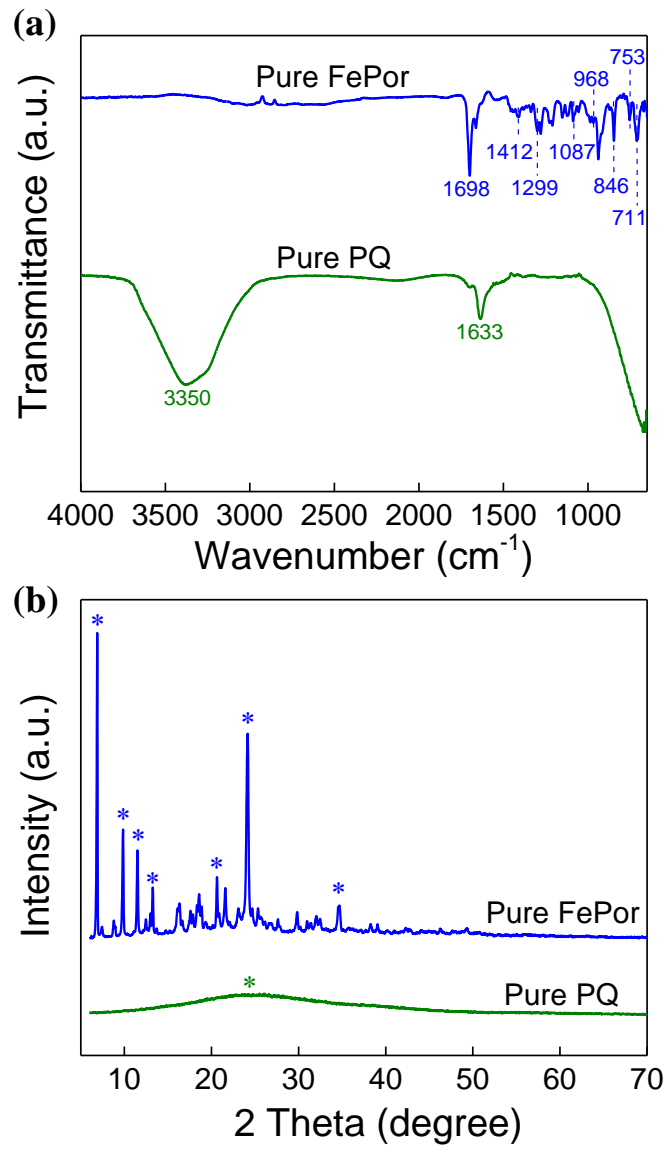

Figure S2. (a) FTIR and (b) XRD spectra of pure FePor and pure PQ. 

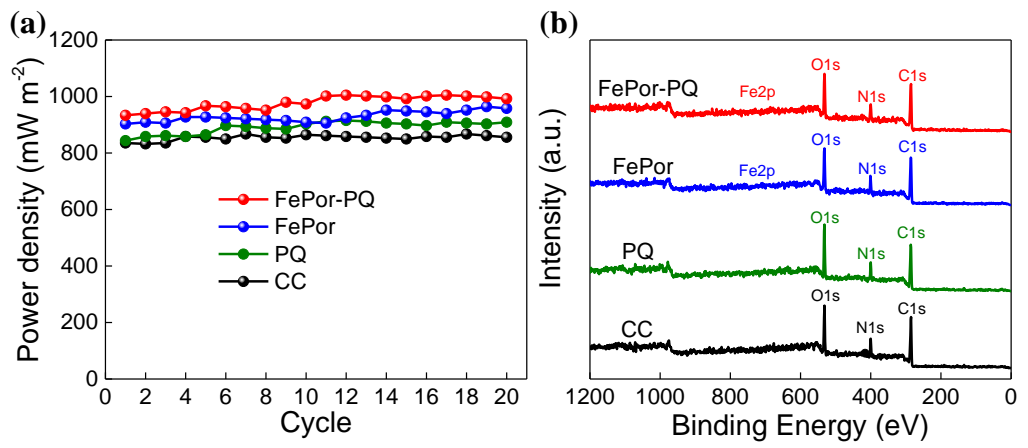

Figure S3. (a) Power density corresponding to the maximum voltage of each cycle for the MFCs with FePor-PQ, FePor, PQ, and CC bioanodes. Voltages were measured with an external resistance of $1000 \Omega$. (b) XPS survey spectra of FePor-PQ, FePor, PQ, and CC bioanodes after operation. 

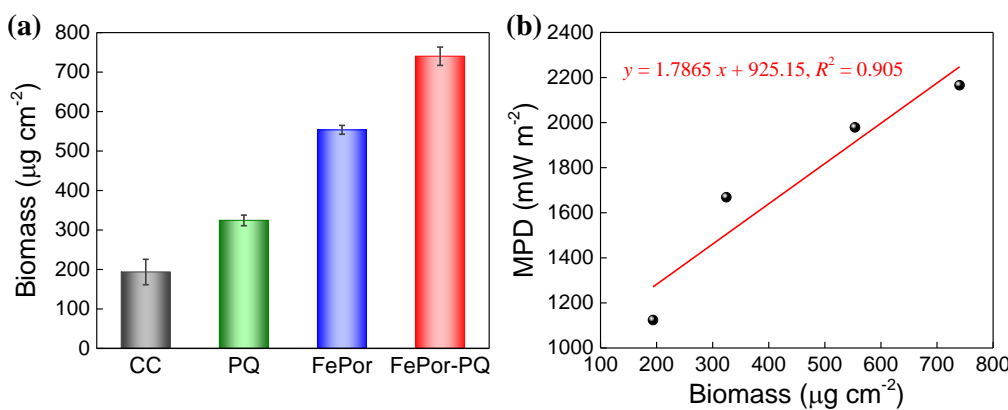

Figure S4. (a) The biomass of CC, PQ, FePor, and FePor-PQ bioanodes. (b) Correlation between biomass and MPD (maximum power density) of different bioanodes. 

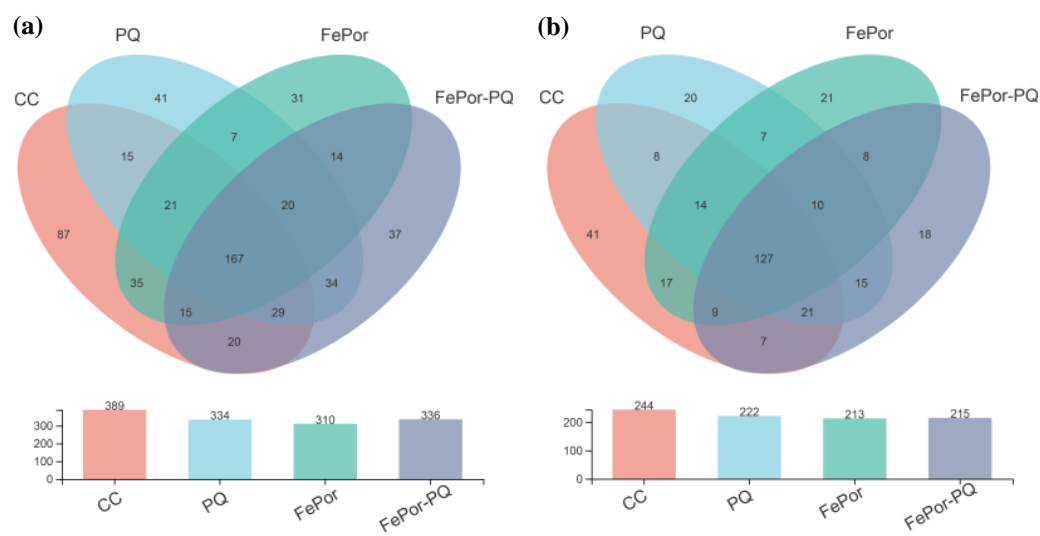

Figure S5. Venn diagrams of different anode biofilms at the (a) OTU level and (b) genus level. 
Table S1. Concentrations of vitamins in solution added to inoculum.

\begin{tabular}{cc}
\hline Composition & $\mathrm{mg} \mathrm{L}^{-1}$ \\
\hline Biotin & 2 \\
Folic acid & 2 \\
Pyridoxine HCl & 10 \\
Riboflavin & 5 \\
Thiamin & 5 \\
Nicotinic acid & 5 \\
Pantothenic acid & 5 \\
B-12 & 0.1 \\
P-aminobenzoic acid & 5 \\
Thioctic acid & 5 \\
\hline
\end{tabular}


Table S2. Concentrations of minerals in solution added to inoculum.

\begin{tabular}{cc}
\hline Composition & $\mathrm{mg} \mathrm{L}^{-1}$ \\
\hline $\mathrm{NTA}$ & 2 \\
$\mathrm{MgSO}_{4}$ & 3 \\
$\mathrm{MnSO}_{4} \cdot \mathrm{H}_{2} \mathrm{O}$ & 0.5 \\
$\mathrm{NaCl}$ & 1 \\
$\mathrm{FeSO}_{4} \cdot 7 \mathrm{H}_{2} \mathrm{O}$ & 0.1 \\
$\mathrm{CaCl}_{2} \cdot 2 \mathrm{H}_{2} \mathrm{O}$ & 0.1 \\
$\mathrm{CoCl}_{2} \cdot 6 \mathrm{H}_{2} \mathrm{O}$ & 0.1 \\
$\mathrm{ZnCl}_{2}$ & 0.13 \\
$\mathrm{CuSO}_{4} \cdot 5 \mathrm{H}_{2} \mathrm{O}$ & 0.01 \\
$\left.\mathrm{AlK} \mathrm{SO}_{4}\right)_{2} \cdot 12 \mathrm{H}_{2} \mathrm{O}$ & 0.01 \\
$\mathrm{H}_{3} \mathrm{BO}_{3}$ & 0.01 \\
$\mathrm{Na}_{2} \mathrm{MoO}_{4}$ & 0.025 \\
$\mathrm{NiCl}_{2} \cdot 6 \mathrm{H}_{2} \mathrm{O}$ & 0.024 \\
$\mathrm{Na}_{2} \mathrm{WO}_{4} \cdot 2 \mathrm{H}_{2} \mathrm{O}$ & 0.025 \\
\hline & \\
\hline & \\
\hline
\end{tabular}


Table S3. Elemental compositions of different electrodes before inoculation and different bioanodes after operation from XPS analysis.

\begin{tabular}{ccccccccc}
\hline & \multicolumn{3}{c}{ Electrodes before inoculation } & \multicolumn{4}{c}{ Bioanodes after operation } \\
\cline { 2 - 8 } Samples & Element C & Element & Element O & Element & Element C & Element & Element O & Element Fe \\
& $($ At\%) & N (At\%) & $($ At\%) & Fe (At\%) & $($ At\%) & N (At\%) & $($ At\%) & $($ At\%) \\
\hline CC & 95.51 & - & 4.49 & - & 65.86 & 12.57 & 21.57 & - \\
PQ & 90.73 & 3.41 & 5.86 & - & 64.92 & 13.05 & 22.03 & - \\
FePor & 92.16 & 1.87 & 5.82 & 0.15 & 67.46 & 13.35 & 18.98 & 0.21 \\
FePor-PQ & 79.75 & 9.59 & 10.49 & 0.17 & 66.43 & 16.30 & 17.05 & 0.22 \\
\hline
\end{tabular}


Table S4. Contents of N and Fe species of different electrodes from XPS analysis.

\begin{tabular}{ccccccccc}
\hline \multirow{2}{*}{ Samples } & \multicolumn{3}{c}{ N species (\%Area) } & \multicolumn{3}{c}{ Fe species (\%Area) } \\
\cline { 2 - 8 } & Pyridinic N & Pyrrolic N & Graphitic N & $\mathrm{Fe}^{2+}\left(2 \mathrm{p}_{3 / 2}\right)$ & $\mathrm{Fe}^{3+}\left(2 \mathrm{p}_{3 / 2}\right)$ & $\mathrm{Satellite}^{2+}$ & $\mathrm{Fe}^{2+}\left(2 \mathrm{p}_{1 / 2}\right)$ & $\mathrm{Fe}^{3+}\left(2 \mathrm{p}_{1 / 2}\right)$ \\
\hline PQ & - & 77.10 & 22.90 & - & - & - & - \\
FePor & 32.24 & 67.76 & - & 26.68 & 23.12 & 16.13 & 20.13 & 13.95 \\
FePor-PQ & 26.73 & 61.85 & 11.42 & 34.60 & 22.60 & 16.01 & 19.09 & 7.70 \\
\hline
\end{tabular}


Table S5. The Ohm resistance $\left(R_{\mathrm{S}}\right)$ and charge transfer resistance $\left(R_{\mathrm{ct}}\right)$ fitted by the equivalent circuit of different anodes before and after inoculation.

\begin{tabular}{ccccc}
\hline \multirow{2}{*}{ Samples } & \multicolumn{2}{c}{ Before inoculation } & \multicolumn{2}{c}{ After inoculation } \\
\cline { 2 - 5 } & $R_{\mathrm{s}}(\Omega)$ & $R_{\mathrm{ct}}(\Omega)$ & $R_{\mathrm{s}}(\Omega)$ & $R_{\mathrm{ct}}(\Omega)$ \\
\hline $\mathrm{CC}$ & 28.8 & 27.0 & 46.6 & 53.2 \\
$\mathrm{PQ}$ & 23.3 & 9.3 & 47.8 & 19.4 \\
FePor & 21.3 & 4.1 & 46.9 & 5.7 \\
FePor-PQ & 18.3 & 4.9 & 36.7 & 5.9 \\
\hline
\end{tabular}


Table S6. Comparison of the FePor-PQ anode in this study and several reported anode materials.

\begin{tabular}{|c|c|c|c|c|c|c|}
\hline Anode material & Anode substrate & Configuration & Influent & Microorganism & Maximum power density $\left(\mathrm{mW} \mathrm{m}^{-2}\right)$ & Refs \\
\hline$\alpha$-FeOOH-NWs & Carbon paper & Double chamber & Lactate & Shewanella loihica PV-4 & 123 & 3 \\
\hline $\mathrm{Fe} @ \mathrm{Fe}_{2} \mathrm{O}_{3} / \mathrm{NCQDs}$ & Carbon cloth & Double chamber & Acetate & Mixed culture & 836 & 4 \\
\hline rGO@PDA/CC & Carbon cloth & Double chamber & Acetate & Mixed culture & 988 & 5 \\
\hline $\mathrm{N}-\mathrm{CNTs} / \mathrm{rGO}$ & Carbon cloth & Double chamber & Lactate & Shewanella putrefaciens CN32 & 1137 & 6 \\
\hline $\mathrm{CC} @ \mathrm{~N}-\mathrm{C} / \mathrm{Fe}_{3} \mathrm{O}_{4}$ & Carbon cloth & Single chamber & Acetate & Mixed culture & 1210 & 7 \\
\hline $\mathrm{Fe}_{2} \mathrm{O}_{3} / \mathrm{PANI} / \mathrm{CC}$ & Carbon cloth & Double chamber & Acetate & Mixed culture & 1503 & 8 \\
\hline Nano-Fe 3 C@PGC & Carbon felt & Single chamber & Acetate & Mixed culture & 1856 & 9 \\
\hline FePor-PQ & Carbon cloth & Double chamber & Acetate & Mixed culture & 2166 & This work \\
\hline
\end{tabular}


Table S7. Species diversity and abundance indices of microbial community in different anode biofilms.

\begin{tabular}{ccccccc}
\hline Samples & OTU num & Shannon $^{\mathrm{a}}$ & Simpson $^{\mathrm{b}}$ & Ace $^{\mathrm{c}}$ & Chao1 $^{\mathrm{c}}$ & Coverage \\
\hline CC & 389 & 2.93 & 0.154 & 492 & 484 & 0.998 \\
PQ & 334 & 2.24 & 0.268 & 518 & 486 & 0.998 \\
FePor & 310 & 1.90 & 0.467 & 401 & 400 & 0.998 \\
FePor-PQ & 336 & 2.12 & 0.341 & 407 & 407 & 0.998 \\
\hline
\end{tabular}

${ }^{a}$ The species diversity index of microbial community focusing on species abundance, a higher number represent more abundance and more diversity.

${ }^{\mathrm{b}}$ The species diversity index of microbial community focusing on species evenness, a higher number represent more evenness but less diversity.

${ }^{\mathrm{c}}$ The species abundance index of microbial community, a higher number represent more abundance. 
Table S8. The relative abundances of several known exoelectrogens in different anode biofilms.

\begin{tabular}{ccccc}
\hline \multirow{2}{*}{ Genus } & \multicolumn{4}{c}{ Relative abundance (\%) } \\
\cline { 2 - 5 } & $\mathrm{CC}$ & $\mathrm{PQ}$ & FePor & FePor-PQ \\
\hline Geobacter & 34.35 & 43.23 & 5.83 & 55.84 \\
Citrifermentans & 16.20 & 0.02 & 67.94 & 0.02 \\
Desulfovibrio & 2.06 & 1.76 & 0.91 & 2.07 \\
Acinetobacter & 4.92 & 0.72 & 1.34 & 4.50 \\
Comamonas & 2.14 & 1.54 & 1.26 & 1.54 \\
Dysgonomonas & 1.77 & 0.36 & 0.83 & 0.14 \\
\hline
\end{tabular}


Table S9. Summarized characteristics of different electrodes.

\begin{tabular}{|c|c|c|c|c|c|c|c|c|c|}
\hline Samples & $\begin{array}{c}\text { Surface } \\
\text { roughness }(\mathrm{nm})\end{array}$ & $\begin{array}{c}\text { Water contact } \\
\text { angle }\left({ }^{\circ}\right)\end{array}$ & $R_{\mathrm{ct}}(\Omega)$ & $\begin{array}{l}\text { EASA } \\
\left(\mathrm{cm}^{2}\right)\end{array}$ & $\begin{array}{c}\text { Maximum } \\
\text { power } \\
\text { density ( } \mathrm{mW} \\
\mathrm{m}^{-2} \text { ) }\end{array}$ & $\begin{array}{l}\text { Peak } \\
\text { current } \\
\text { density } \\
\left(\mathrm{A} \mathrm{m}^{-2}\right)\end{array}$ & $\begin{array}{l}\text { Heterogeneous } \\
\text { electron transfer } \\
\text { rate constant } \\
\quad\left(\mathrm{s}^{-1}\right)\end{array}$ & $\begin{array}{l}\text { Contribution } \\
\text { ratios of fast } \\
\text { kinetic process } \\
\text { (\%) }\end{array}$ & $\begin{array}{l}\text { Biomass } \\
\left(\mu \mathrm{g} \mathrm{cm}^{-2}\right)\end{array}$ \\
\hline $\mathrm{CC}$ & $63.1 \pm 3.0$ & $139.4 \pm 5.5$ & 27.0 & 4.37 & 1124.0 & 0.23 & 1.26 & $19.54-27.75$ & $193.6 \pm 32.2$ \\
\hline PQ & $63.4 \pm 2.9$ & $\sim 0$ & 9.3 & 6.30 & 1668.8 & 0.32 & - & - & $324.3 \pm 13.4$ \\
\hline FePor & $85.4 \pm 4.9$ & $118.2 \pm 2.2$ & 4.1 & 6.70 & 1978.9 & 0.53 & - & - & $553.7 \pm 11.1$ \\
\hline FePor-PQ & $118.3 \pm 9.5$ & $48.5 \pm 7.0$ & 4.9 & 7.22 & 2165.7 & 1.07 & 2.15 & $45.22-56.62$ & $741.2 \pm 23.2$ \\
\hline
\end{tabular}




\section{References}

(1) Laviron, E. General Expression of the Linear Potential Sweep Voltammogram in the case of Diffusionless Electrochemical Systems. J. Electroanal. Chem. 1979, 101 (1), 19-28.

(2) Yao, B.; Chandrasekaran, S.; Zhang, H.; Ma, A.; Kang, J.; Zhang, L.; Lu, X.; Qian, F.; Zhu, C.; Duoss, E. B.; Spadaccini, C. M.; Worsley, M. A.; Li, Y. 3D-Printed Structure Boosts the Kinetics and Intrinsic Capacitance of Pseudocapacitive Graphene Aerogels. Adv. Mater. 2020, 32 (8), 1906652.

(3) Xian, J.; Ma, H.; Li, Z.; Ding, C.; Liu, Y.; Yang, J.; Cui, F. Alpha-FeOOH Nanowires Loaded on Carbon Paper Anodes Improve the Performance of Microbial Fuel Cells. Chemosphere 2021, 273, 129669.

(4) Farahmand Habibi, M.; Arvand, M.; Sohrabnezhad, S. Boosting Bioelectricity Generation in Microbial Fuel Cells Using Metal@Metal Oxides/Nitrogen-Doped Carbon Quantum Dots. Energy 2021, 223, 120103.

(5) Liu, H.; Zhang, Z.; Xu, Y.; Tan, X.; Yue, Z.; Ma, K.; Wang, Y. Reduced Graphene Oxide@Polydopamine Decorated Carbon Cloth as an Anode for a High-Performance Microbial Fuel Cell in Congo Red/Saline Wastewater Removal. Bioelectrochemistry 2021, 137, 107675.

(6) Wu, X.; Qiao, Y.; Shi, Z.; Tang, W.; Li, C. M. Hierarchically Porous N-Doped Carbon Nanotubes/Reduced Graphene Oxide Composite for Promoting Flavin-Based Interfacial Electron Transfer in Microbial Fuel Cells. ACS Appl. Mater. Interfaces 2018, 10 (14), 11671-11677.

(7) Wang, Y.; Liu, C.; Zhou, S.; Hou, R.; Zhou, L.; Guan, F.; Chen, R.; Yuan, Y. Hierarchical N-Doped $\mathrm{C} / \mathrm{Fe} 3 \mathrm{O} 4$ Nanotube Composite Arrays Grown on the Carbon Fiber Cloth as a Bioanode for HighPerformance Bioelectrochemical System. Chem. Eng. J. 2021, 406, 126832.

(8) Li, M.; Zhou, S. $\alpha$-Fe2O3/Polyaniline Nanocomposites as an Effective Catalyst for Improving the Electrochemical Performance of Microbial Fuel Cell. Chem. Eng. J. 2018, 339, 539-546.

(9) Hu, M.; Li, X.; Xiong, J.; Zeng, L.; Huang, Y.; Wu, Y.; Cao, G.; Li, W. Nano-Fe3C@PGC as a Novel Low-Cost Anode Electrocatalyst for Superior Performance Microbial Fuel Cells. Biosens. Bioelectron. 2019, 142, 111594. 